\title{
Modeling of Initial Detonation-mode Acceleration in Pulsed Plasma and Magnetoplasmadynamic Thrusters
}

\author{
Kurt A. Polzin* and Christine M. Greve ${ }^{\dagger}$ \\ NASA-Marshall Space Flight Center, Huntsville, AL 35812
}

\begin{abstract}
Gas-fed electromagnetic pulsed plasma accelerators operate by discharging electrical energy into a gas, subsequently ionizing and electromagnetically accelerating propellant. Many efforts to model pulsed accelerators have assumed that the discharge is either short and completely transient, accelerating the gas like a shock by entraining it in a moving current sheet, or that the discharge is relatively long, establishing a stable quasi-steady current distribution through which plasma flows and is accelerated. This idealization encounters problems when thrusters possess some qualities associated with both short and long-pulse-length thrusters. To capture all possible scenarios, a model is presented based upon the idea that all pulsed plasma accelerators first form an accelerating current sheet (detonation mode accelerator) and then, depending upon the pulse length and the manner in which the plasma reaches the thruster exit, it can transition to the quasi-steady acceleration configuration (deflagration mode accelerator). In the present work the detonation mode is investigated, varying controllable parameters to determine their effects on the plasma acceleration process. The primary driver affecting current sheet acceleration is the amount of gas that the the plasma encounters and entrains as it moves towards the thruster exit. The amount of neutral gas the plasma entrains affects the time it takes the plasma to reach the end of the accelerator and changes the corresponding electrical discharge parameters at the end of detonation mode acceleration.
\end{abstract}

\section{Introduction}

$\mathrm{P}$ ULSED plasma accelerators typically operate by accelerating a plasma by the Lorentz body force. This force is generated when energy stored in a capacitor bank is discharged into a gas, ionizing the gas and generating a selfinduced magnetic field that interacts with the current. The present work focuses on two types of thrusters that follow this general operating scheme: the gas-fed pulsed plasma thruster (PPT) ${ }^{1}$ and the quasi-steady magnetoplasmadynamic (MPD) accelerator ${ }^{2}$ - and is a continuation of previous work to analyze these types of thrusters. ${ }^{3}$

The gas-fed pulsed plasma accelerator is generally represented as a completely transient device discharging in $\sim 1-10 \mu \mathrm{s}$. The plasma current sheet forms at the breech of the thruster and propagates forward in what is known as a 'snowplow' or 'detonation' mode due to its entrainment of the gas it encounters as it accelerates down the thruster. The representation of this motion is akin to strong detonation shockwave propagation, and so it is often referred to as operation in the 'detonation mode.' This acceleration ceases when either the current sheet reaches the end of the thruster or the current in the circuit reverses direction and deprives the initial sheet of additional acceleration. In contrast, the quasi-steady MPD accelerator has a pulse length of $\sim 1 \mathrm{~ms}$ or longer that is typically maintained at an approximately constant level throughout the discharge by a pulse-forming network (PFN) of capacitors. An initial transient discharge that is short relative to the entire pulse gives way to a 'quasi-steady' operating mode, where ionized gas passes through a stationary current channel in which the plasma experiences electromagnetic acceleration. This is termed 'deflagration mode' operation owing to the similarities to deflagration waves found in shock and combustion physics. The MPD boasts high thrust density and efficiency (up to 50\%) as well as low electrode erosion rates in comparison to the PPT that has efficiencies in the teens.

There are examples in the literature where a pulsed thruster does not fit particularly well into either category, such as the Coaxial High ENerGy (CHENG) thruster developed by Cheng. ${ }^{4}$ The CHENG thruster operates on the $\sim 10$ $\mu$ s timescales of pulsed plasma thrusters, but claims the high thrust density, high efficiency and low electrode erosion rates more consistent with the deflagration mode of acceleration. Another set of thrusters that deviate from expected behavior are the gas-fed PPTs by Ziemer, et al. ${ }^{5,6}$ In that work, two separate regimes of thruster performance were

* Propulsion Research and Technology Applications Branch. Associate Fellow AIAA.

${ }^{\dagger}$ Presently: Graduate Research Assistant, Texas A\&M University, College Station, TX. Student Member AIAA. 
identified. The regime at higher mass bits (termed Mode I in that work) possessed relatively constant thrust efficiency (ratio of jet kinetic energy to input electrical energy) as a function of mass bit. In the second regime at very low mass bits (termed Mode II), the efficiency increased with decreasing mass bit.

The CHENG thruster, ${ }^{4}$ which didn't cleanly fit into the standard PPT/MPD classification scheme, and the rapid change in gas-fed PPT operational modes identified by Ziemer, et al. ${ }^{5,6}$ have motivated previous investigations and serve as the prime motivation for the present work. Previous efforts by Poehlmann et al. ${ }^{7}$ and by Sitaraman and Raja $^{8}$ sought to understand the performance of the CHENG thruster and the Mode I/Mode II performance in PPTs by modeling the acceleration of the plasma using the Hugoniot Relation. The detonation and deflagration modes were treated as distinct solution sets to the relevant conservation laws across a shock. The ultimate conclusion of these works was that the thrusters could be made to operate in one mode or the other by varying the values of different parameters.

We differ from previous modeling works by postulating that all electromagnetic pulsed plasma accelerators can be understood by considering and accurately modeling the entire time history of the discharge. ${ }^{3}$ Our modeling of the initial stage of the discharge in all cases treats the plasma as a current sheet forming at the breach of the accelerator and moving towards the exit as a detonation wave. If the current sheet reaches the exit of the accelerator before the discharge is completed, the current sheet transitions to a deflagration mode-type discharge like those found in quasi-steady MPD thrusters. By treating the initial stage of the thruster discharge as a detonation-like plasma sheet and allowing the plasma the opportunity to transition to a deflagration mode, we aim to demonstrate that the overall acceleration of the plasma is dependent upon the behavior of the initial transient phase and the relative lengths of the transient and quasi-steady modes of operation.

In the present work, we specifically examine the detonation mode portion of the acceleration process. Modeling this process is the first step in the overall modeling of a pulse electromagnetic accelerator as the detonation mode end state serves as the initial set of conditions for deflagration-mode acceleration. There are various parameters that can be controlled or that vary in this process, and we study several including the delay between the start of gas injection and the initiation of the current sheet and the effect on current acceleration owing to variations in the mass flow rate, the percentage of gas that is actually entrained when it encounters a current sheet, and the amount of gas shed by a current sheet as it moves towards the thruster exit.

In Sect. II we describe the governing equations that will be used to model pulsed electromagnetic thrusters in this and subsequent works. A discussion of the test cases performed to ensure the conservation of mass and momentum can be found in Sect. III. Following in Sect. IV are modeling results obtained when varying different parameters of the problem. In Sect. V, we discuss the results and their support of the proposed hypothesis for pulsed electromagnetic acceleration.

\section{Governing Equations}

The governing equations can be separated into the categories of those affecting the neutral gas motion, those capturing the accelerating plasma current sheet (detonation mode), and those modeling the acceleration of the gas imparted by the stationary plasma upon reaching the end of the thruster (deflagration mode). The fluid solver and current sheet solver are required to be solved on different time scales due to the relative speeds of the objects of interest. The fluid solver uses an Eulerian frame of reference which is overlaid by a pulsed plasma thruster current sheet solver in the Lagrangian frame of reference. For completeness, in this section we repeat the governing equation set found in Ref. [3]. The present work assumes a thruster based upon the coaxial accelerator studied by Poehlmann et al. ${ }^{7}$ possessing properties given in Table 1 .

\section{A. Fluid Equations}

While the problem at hand will be solved in one dimension, in general, the conservation equations governing the evolution of a species of a fluid are given as

$$
\begin{aligned}
\frac{\partial \rho}{\partial t}+\nabla \cdot(\rho \mathbf{u}) & =0 \\
\frac{\partial(\rho \mathbf{u})}{\partial t}+\nabla \cdot(\rho \mathbf{u u}+\overline{\bar{P}}) & =\nabla \cdot \overline{\bar{\tau}}_{\text {vis }}+\mathbf{f}_{\text {ext }} \\
\frac{P}{\rho^{\gamma}} & =\text { constant }
\end{aligned}
$$


Table 1: Properties for the Thruster Simulation

\begin{tabular}{l|r}
\hline Thruster Length & $23 \mathrm{~cm}$ \\
Inner electrode diameter & $5 \mathrm{~mm}$ \\
Outer electrode diameter & $5 \mathrm{~cm}$ \\
Capacitance $C$ & $112 \mu F$ \\
Stray inductance $L_{0}$ & $50 \mathrm{nH}$ \\
Stray resistance $R_{e}$ & $5 \mathrm{~m} \Omega$ \\
Plasma resistance $R_{p}$ & $2.5 \mathrm{~m} \Omega$ \\
Initial charge voltage & $1000 \mathrm{~V}$ \\
Inlet gas temperature & $298 \mathrm{~K}$ \\
\hline
\end{tabular}

where $\overline{\bar{P}}$ is the pressure tensor possessing a scalar value of $P, \overline{\bar{\tau}}_{\text {vis }}$ denotes the viscous stress tensor, $\mathbf{f}_{e x t}$ is an external body force applied to the control volume, and $\gamma$ is the ratio of specific heats. Conservation of mass Eq. (1a) and conservation of momentum Eq. (1b) are used to calculate the evolution of neutral fluid density and momentum at every point within the domain. The first terms on the left-hand side of Eqs. (1a) and (1b) represent the time rate of change in a control volume of the conserved quantities of density $\rho$ and momentum density $\rho \mathbf{u}$, while the divergence terms on the left-hand side represent the net flux of mass and momentum density, respectively, into the control volume. Equation (1a) is set equal to zero as there is no gas source or sink included in the model. We shall typically ignore the viscous effects for the present work. A one-dimensional isentropic "closure" approximation is used to solve for the evolution of the pressure within the domain.

The solver includes a single ghost cell at the beginning and end of the modeled 1-D channel. The initial inlet ghost cell is used to set the mass flow rate into the actual domain. This is accomplished by setting pressure in the oncoming fluid, calculating the initial ghost cell density using an assumed temperature and an ideal gas equation of state, and then setting the velocity to yield a desired inlet mass flow rate $\dot{m}=\rho u A$. To ensure that the mass flux into the domain is equal to $\dot{m}$, the values of $\rho, u$, and $P$ in the first cell of the domain are set equal to the ghost cell values. The outlet of the domain employs a Neumann-type boundary condition for the ghost cell. By copying the values of the last cell into the ghost cell, a zero gradient is maintained across the last cell boundary.

The values of mass density, momentum density, and pressure are stored at the cell centers, and using linear interpolation, values at the cell boundaries can be found. To generate a solution, the cell-centered values from the previous time step are used to calculate the mass and momentum fluxes into and out of each cell. After the densities are updated, the cell-centered pressures are updated using Eq. (1c).

The sheet wake consists of the gas not entrained by the current sheet, which will be moving at a slow speed, and the gas shed from the current sheet at every time step, which will be shed with the speed of the current sheet at that particular time step. These two fluids interact through their pressure terms as they each contribute a partial pressure of the entire cell pressure. There is also collisional drag between the two fluids since they are moving at disparate speeds. Momentum is transferred through collisions between the two species according to

$$
f_{\text {ext }}= \pm \frac{m_{12} n_{1} n_{2} d^{2}}{2}\left(u_{1}-u_{2}\right) \triangle t\left(\frac{8 \pi k T}{m_{12}}\right)^{1 / 2}
$$

In this equation, the subscript 1 denotes the fast fluid species while the subscript 2 denotes the slow species. The equation is positive for the "slow" species while negative for the "fast" species as the transfer of momentum requires.

\section{B. Plasma Sheet Acceleration Equations}

The differential equations governing the current sheet motion as it moves through the already-injected neutral gas are given below. The present model uses a simple $R L C$-circuit though it can be readily altered to accommodate other electrical pulse sources such as pulse-forming networks. 


$$
\begin{aligned}
\frac{d V}{d t} & =-\frac{I}{C} \\
\frac{d I}{d t} & =\frac{V-I\left[\left(R_{e}+R_{p}\right)+L_{1} v_{z}\right]}{\left(L_{0}+L_{1} z\right)} \\
\frac{d z}{d t} & =v_{z} \\
\frac{d v_{z}}{d t} & =\left(\frac{L_{1} I^{2}}{2}-v_{z}{ }^{2} \lambda_{e} A \rho(z)\right) / m \\
\frac{d m}{d t} & =v_{z} A \rho(z) \frac{\lambda_{e}}{\left(1+\nu_{\text {loss }}\right)}
\end{aligned}
$$

Equations (3a) and (3b) describe the solution to the voltage $V$ and current $I$ in an electrical circuit possessing an inductive element moving with the axial velocity $v_{z}$. The current sheet position $z$, velocity $v_{z}$, and accumulated mass $m$ are modeled using Eqs. (3c), (3d), and (3e), respectively. The initial or 'stray' inductance of the circuit is given as $L_{0}$ while the inductance per unit length along the accelerator electrodes is $L_{1}$. The stray circuit resistance and plasma resistance are $R_{e}$ and $R_{p}$, respectively. The first term on the right-hand side of Eq. (3d) represents the Lorentz body force accelerating the plasma while the second term is the loss of sheet momentum owing to the inelastic acceleration of gas entrained by the current sheet up to the sheet speed. The value of $\rho(z)$ is the density of the gas encountered by the current sheet, and is generally going to be the gas density resulting from the evolution of Eq. (1a) for the initiallyinjected gas species. The cross-sectional area of the thruster exhaust is $A$ and the current sheet permeability term $\lambda_{e}$ is a parameter that accounts for the fraction of neutral gas that is actually entrained by the current sheet. The term $\nu_{\text {loss }}$ in the denominator can also be varied parametrically to adjust the mass lost out of the current sheet during each time step.

\section{Stationary Discharge Plasma Acceleration Equations}

While solutions for the deflagration mode are not given in the present work, for completeness we describe the equations that will be used to solve for this mode when it is included in the model. When the plasma reaches the end of the electrodes, it is treated as a stationary plasma imparting electromagnetic acceleration to gas that moves through it. In this case a pulsed discharge is still occurring but the plasma is no longer moving so there is no variable inductance term or plasma sheet motion in the equation set. The voltage equation remains the same as Eq. (3a) but the current equation changes to yield the circuit equation set

$$
\begin{aligned}
\frac{d V}{d t} & =-\frac{I}{C} \\
\frac{d I}{d t} & =\frac{V-I\left(R_{e}+R_{p}\right)}{\left(L_{0}+L_{1} l\right)}
\end{aligned}
$$

where $l$ is the length of the accelerator. The end state of Eqs. (3a) and (3b) are used as the initial conditions for Eqs. (4a) and (4b). In future work, the solution of these equations will used to calculate the electromagnetic body force on the fluid passing through the stationary plasma. This force will be applied to the fluid through the $\mathbf{f}_{\text {ext }}$ term of Eq. (1b), the magnitude of which may be calculated as

$$
f_{\text {ext }}=\frac{L_{1} I^{2}}{2}
$$

The current is solved on a different time scale than the fluid solver and so must be averaged to find a force density to apply per unit of fluid solver time.

$$
\left\langle\mathrm{f}_{\text {ext }}\right\rangle=\frac{1}{\left(t_{2}-t_{1}\right)} \int_{t_{1}}^{t_{2}} \mathrm{f}_{\text {ext }}(t) d t
$$

where $\left\langle\mathrm{f}_{\mathrm{ext}}\right\rangle$ is the average value between $t_{1}$ and $t_{2}$ which denote the start and end of a fluid solver time step. 


\section{Test Cases}

We describe a series of test cases that were solved to ensure that various conserved properties remained conserved throughout the solution process.

\section{A. Gas Injection}

The simplest test was performed to ensure that "injected" mass was conserved as it propagated in the computational domain. In the first instance, gas was injected for a given period of time, evolving to the density distribution shown in Fig. 1a where no gas had yet exited the domain. The gas distribution was integrated numerically, with the result in Fig. 1b showing the integrated gas as a fraction of the total mass injected. We observe that the fraction approaches 1 as the exit is approached, indicating mass conservation. This check was performed for different mass flow rates and different domain sizes to demonstrate that mass remained conserved in all instances. The same method was used to determine that momentum was being conserved in the numerical scheme.

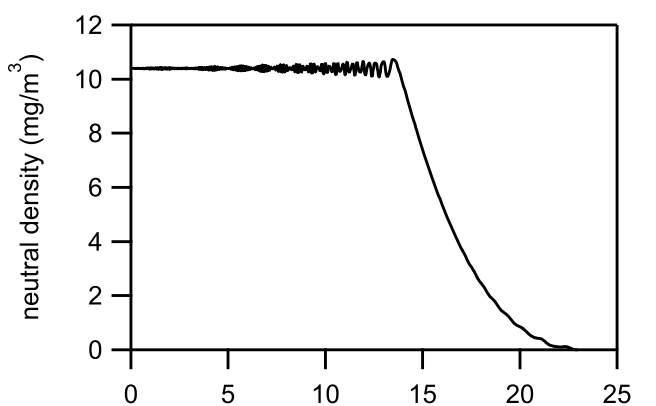

a)

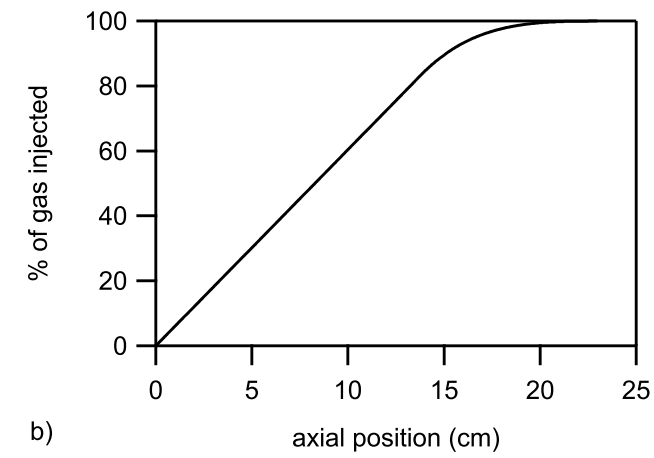

Figure 1: a) Neutral gas density at a mass flow rate of $8 \mathrm{mg} / \mathrm{s}$ for an injection time of $410 \mu \mathrm{s}$, and b) the integrated gas in the domain as a percentage of the total gas injected from $t=0$ to the instant shown.

We stopped the gas injection solver at different instances in time to see how long it took for the gas front to advance within the channel. Density distributions for different elapsed injection times are presented in Fig. 2a. Computing the thermal speed as $v_{t h}=\sqrt{8 k T /\left(\pi m_{\mathrm{Ar}}\right)}$ using a temperature of $298 \mathrm{~K}$, we estimate that the channel transit time is just over $0.55 \mathrm{~ms}$, which matches well with computed time-evolution of density. Shown in Fig. $2 \mathrm{~b}$ are channel density distributions at a single instant in time for varying inlet mass flow rate. The front advances at the same rate in all instances, and the steady density value behind the front scales linearly with flow rate, as expected.

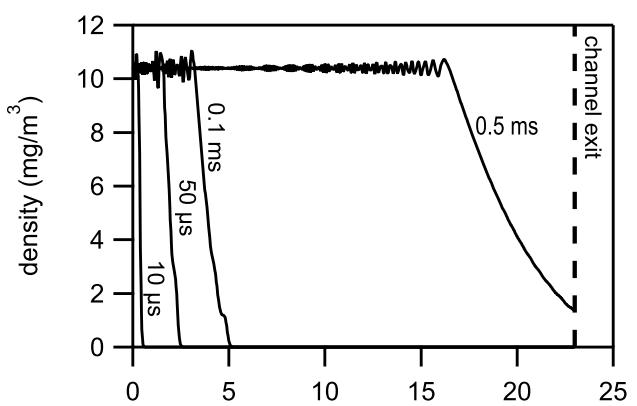

a)

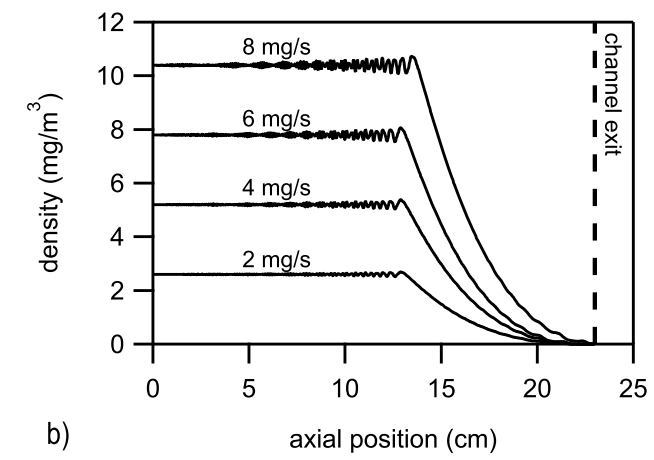

Figure 2: Neutral gas density in the channel a) at different instances of time for a mass flow rate of $8 \mathrm{mg} / \mathrm{s}$ and b) at an injection time of $410 \mu$ s for different mass flow rates as shown. 


\section{B. Plasma Current Sheet Acceleration}

The plasma current sheet acceleration and entrainment efficiency terms were validated by using a saved gas injection case as a starting point for the distribution of neutral density and momentum in the channel. The gas injection mass flow rate was $8 \mathrm{mg} / \mathrm{s}$ and delay time $t_{\text {delay }}$ between the start of neutral gas injection and current sheet initiation for these test cases was $410 \mu \mathrm{s}$. This time was selected because it occurs just before any gas has exited the thruster channel, as demonstrated in Fig. $2 b$.

The current sheet trajectory was solved while varying the mass entrainment coefficient $\lambda_{e}$ from 0.2-1.0 with a constant sheet mass loss coefficient $\nu_{\text {loss }}=0$, and while varying $\nu_{\text {loss }}$ from $0-1$ with a constant $\lambda_{e}=1.0$. When $\lambda_{e}$ is varying, it is a relatively straightforward exercise to compare the mass in the current sheet to the total neutral mass in the channel as a function of time and plot this percentage in the manner shown in Fig. 3a. We see that the percent of encountered mass entrained matches the value of $\lambda_{e}$ when the current sheet reaches the end of the channel at $z=23$ $\mathrm{cm}$. We also observe that since the drag resistance on the current sheet scales with the mass entrainment coefficient (see Eq. (3d)), the sheet reaches the end of the thruster channel much more quickly as $\lambda_{e}$ is reduced. When $\nu_{\text {loss }}$ is increased, the current sheet sheds already-entrained mass, reducing the overall mass of the current sheet seen in Fig. $3 \mathrm{~b}$. The final mass of the current sheet is half of the total mass the sheet encounters when $\nu_{\text {loss }}=1$, which is the expectation based upon Eq. (3e). Interestingly, the time it takes for the current sheet to reach the end of the channel is only slightly less for increasing $\nu_{\text {loss }}$. We shall discuss this result more in the next section.

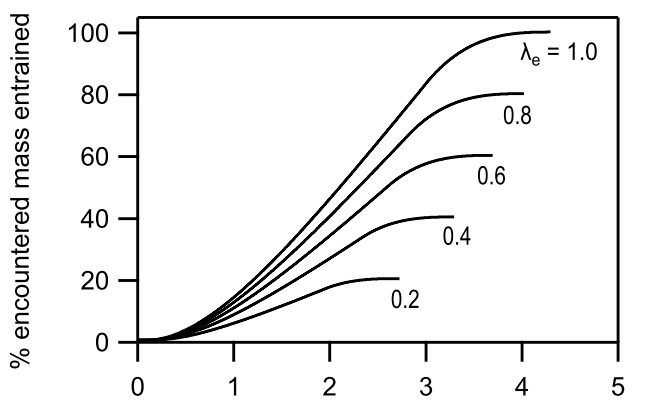

a)

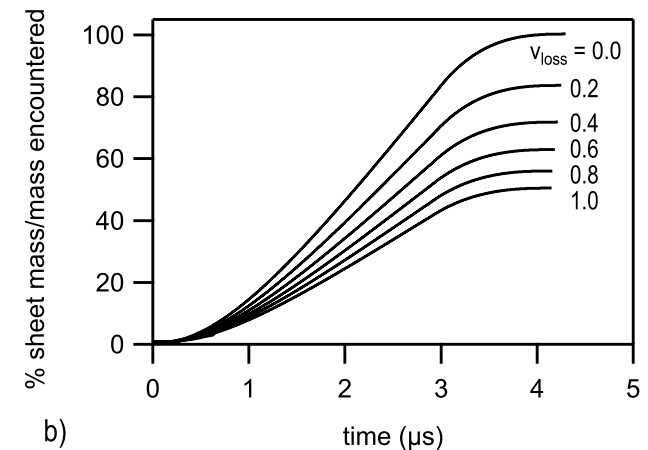

b)

Figure 3: a) Percent of encountered gas entrained in the current sheet as a function of $\lambda_{e}$ as shown, with $\nu_{\text {loss }}=0$, and for different values of $\nu_{\text {loss }}$ as shown, with $\lambda_{e}=1.0$. The delay time $t_{\text {delay }}$ between the start of gas injection and current sheet initiation is $410 \mu \mathrm{s}$. Each curve ends when the current sheet reaches the end of the channel at $z=23 \mathrm{~cm}$.

\section{Current Sheet Wake}

After the current sheet passes a location, there can be mass remaining in the wake. This can be either because not all the mass was entrained by the current sheet $\left(\lambda_{e}<1\right)$ or because the current sheet shed mass initially entrained by it $\left(\nu_{\text {loss }}>0\right)$. To verify mass conservation, we first integrated the mass in the domain before current sheet initiation. This mass was compared to the sum of the mass entrained in the current sheet, the mass of gas that was not entrained by the current sheet, and the mass of gas shed by the current sheet to ensure that all mass was accounted at the end of the current sheet acceleration stage.

\section{Modeling Results}

In this section, we present and discuss results obtained by solving the fluid and accelerating plasma sheet parts of the problem to show how varying different controllable parameters affect the detonation mode phase of the problem. Both the fluid end state and the pulsed discharge state when the current sheet reaches the end of the thruster channel form the initial condition set for the stationary discharge plasma acceleration phase of the solution. 


\section{A. Gas Injection}

We presented data in Fig. 2a showing the injected neutral gas density in the channel for a constant mass flow rate and different instances in time (measured from $t=0$ when gas injection begins) and channel gas densities in Fig. $2 \mathrm{~b}$ for different mass flow rates taken at a specific instant in time. These data form the basis of the neutral gas density $\rho(z)$ into which the current sheet advances once it is initiated. To minimize the oscillations in the density observed in the figures, a CFL condition $\Delta x / \Delta t=v_{f} / 0.0001$ is used, where $v_{f}$ is the fastest fluid velocity in the computational domain.

\section{B. Current Sheet Trajectories}

Plasma current sheet solutions were generated for a number of different cases to determine the effect of different parameters on detonation-mode acceleration. What follows is a presentation of the various solutions and a discussion of the data. For all cases, the plasma sheet detonation-mode calculations are halted when the plasma reaches the end of the channel at $z=23 \mathrm{~cm}$.

The neutral gas into which the plasma sheet is advancing is controlled by the mass flow rate of gas. Presented in Fig. $4 \mathrm{a}$ are data for $\lambda_{e}=1.0$ and $\nu_{\text {loss }}=0$ showing the current sheet trajectories as a function of time as the delay time $t_{\text {delay }}$ between the start of gas injection and current sheet initiation is varied. The values of $t_{\text {delay }}$ correspond to the neutral fluid gas density conditions presented in Fig. 2a. We observe that the plasma reaches the end of the channel much faster when $t_{\text {delay }}$ is reduced since there is no resistance to plasma acceleration once the current sheet moved beyond the injected neutral gas. In fact, until the accelerating plasma sheet moves beyond the gas, it is always snowplowing into the same gas density and so all the curves follow the same trajectory until they get beyond the constant fluid density region. Another interesting effect is observed in Fig. 4b, which shows the discharge current values as a function of $t_{\text {delay }}$. We observe that they all follow the same initial current waveform, but as the sheet moves past the constant fluid density region, the current crests and begins to decrease. Physically, with reduced neutral gas resistance to current sheet motion, it accelerates much more rapidly towards the exit, but in doing so the inductance, and consequently the impedance, presented to the circuit by the thruster increases. As a consequence, for smaller $t_{\text {delay }}$ the initial current sheet is ejected much more rapidly and there remains significantly more voltage on the capacitor available to drive the stationary discharge plasma acceleration process. We demonstrated in [3] that these results also held as the neutral mass flow rate was reduced.
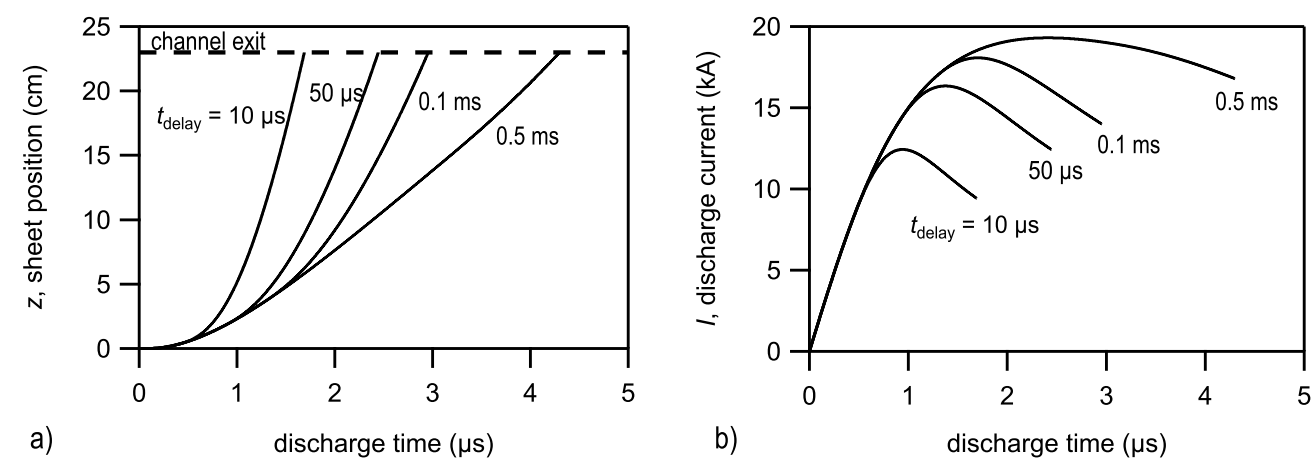

Figure 4: Plots of a) current sheet position and b) discharge current as a function of time as the delay time $t$ delay between the start of gas injection and current sheet initiation is varied while $\lambda_{e}=1.0$ and $\nu_{\text {loss }}=0$.

In Fig. 5 we present data for $t_{\text {delay }}=410 \mu$ s and $\nu_{\text {loss }}=0$ showing the current sheet trajectory and discharge current as a function of time for varying values of $\lambda_{e}$. These data correspond to the graph of percent of encountered mass entrained as a function of $\lambda_{e}$ presented earlier in Fig. 2a. Changing the mass entrainment fraction affects the trajectory of the the current sheet in a manner similar to adjusting $t$ delay since both reduce the drag extered by neutral gas entrained by the current sheet.

In Fig. 6 we present data for $t_{\text {delay }}=410 \mu$ s and $\lambda_{e}=1.0$ showing the current sheet trajectory and discharge current as a function of time for varying values of $\nu_{\text {loss }}=0$. These data correspond to the graph of percent of encountered mass entrained as a function of $\nu_{\text {loss }}$ presented earlier in Fig. 2b. The data show that as $\nu_{\text {loss }}$ is increased from 0 to 1 , the final current sheet mass is reduced by a factor of 2 . The interesting part is that the time to reach the 

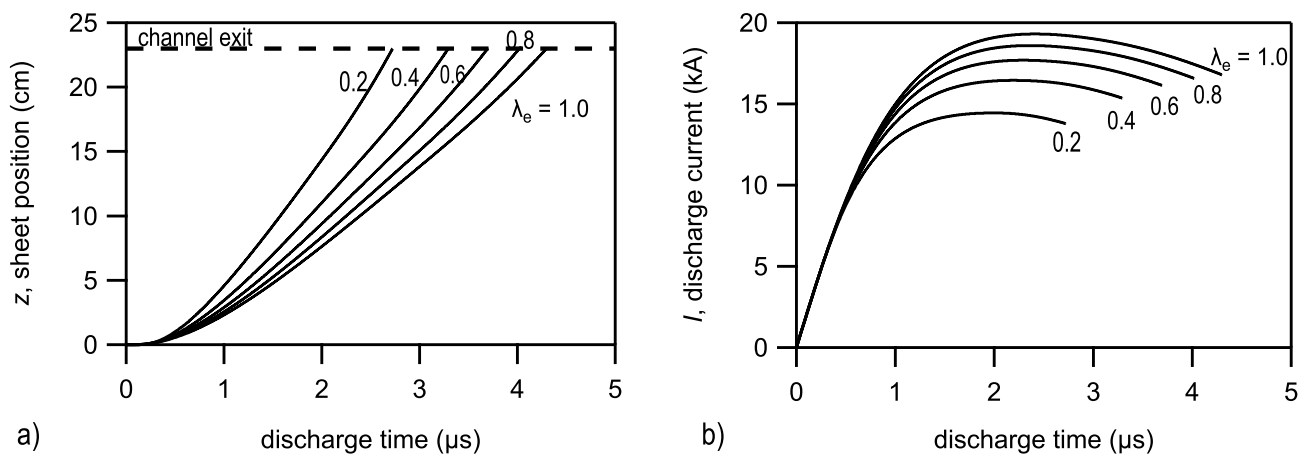

Figure 5: Plots of a) current sheet position and b) discharge current as a function of $\lambda_{e}$ as shown, with $t_{\text {delay }}=410$ $\mu$ s and $\nu_{\text {loss }}=0$.

end of the channel is only slightly decreased for increasing $\nu_{\text {loss }}$, even as the current sheet mass is much smaller. We find that the velocity of the sheet is slightly greater for increased $\nu_{\text {loss }}$. Exploring Eq. (3d) we note that this slightly larger velocity increases the resistive drag applied to the current sheet as it encounters neutral gas. In addition, because the sheet is moving a bit faster axially, the impedance presented to the circuit by the thruster increases reducing the discharge current slightly. For the specific case where $\nu_{\text {loss }}$ is increased from 0 to 1 , the overall loss in electromagnetic acceleration $\left(\propto I^{2}\right)$ and increase in neutral entrainment drag $\left(\propto v_{z}^{2}\right)$ are such that the acceleration force is decreased by a factor of roughly 2 . When divided by the current sheet mass, which is a factor of 2 smaller when $\nu_{\text {loss }}$ is increased from 0 to 1 , the net result is approximately the same acceleration profile for both cases.
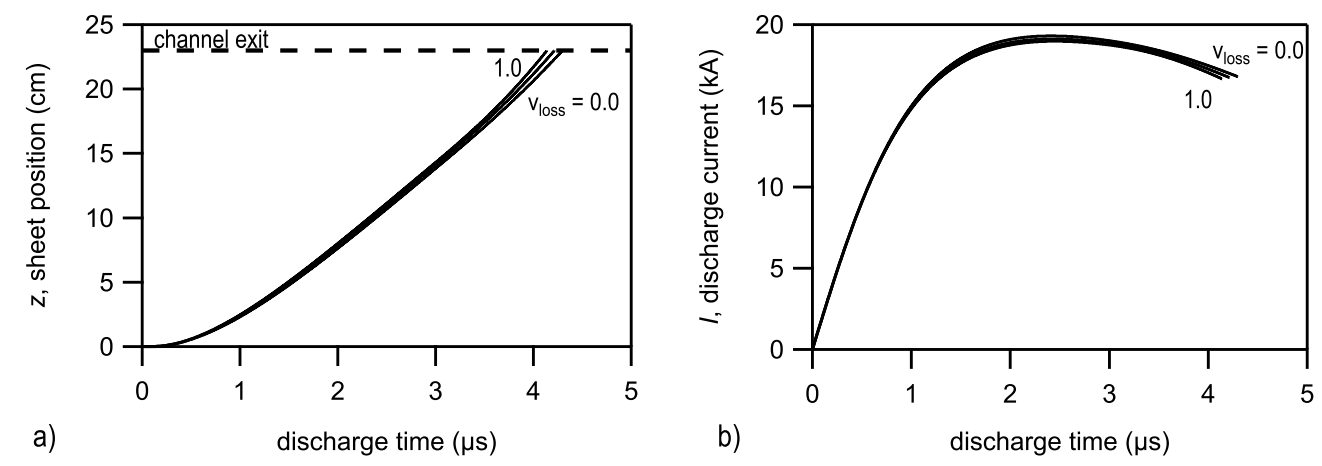

Figure 6: Plots of a) current sheet position and b) discharge current as a function of $\nu_{\text {loss }}$ as shown, with $t_{\text {delay }}=410$ $\mu \mathrm{s}$ and $\lambda_{e}=1.0$.

\section{Discussion}

We hypothesized that we could better understand the trends in pulsed plasma accelerator data by treating the initial stage of the thruster discharge as a detonation-mode accelerating plasma sheet and then allowing the plasma the opportunity to transition to a deflagration mode. Specifically, we believed that the behavior during the initial transient phase and the relative lengths of the transient and quasi-steady modes of operation held the key to understanding pulsed thruster data that didn't neatly fit into either the PPT or MPD modes of operation. While we have not yet attempted the solution of the stationary discharge plasma acceleration problem, the neutral gas injection and detonation-mode acceleration solutions that form the basis of the stationary discharge initial conditions give insight into the validity of our hypothesis.

The CHENG thruster ${ }^{4}$ operated on short $\sim 10 \mu$ s timescales but had high thrust density, high efficiency and low electrode erosion rates more consistent with the deflagration mode of acceleration. The CHENG thruster was also 
operated in a 'switchless' mode, meaning that the discharge would initiate as soon as neutral gas was injected into the accelerator. This means that $t_{\text {delay }}$ was very short and, like shown in Fig. 4, the neutral gas would likely provide very little resistance to the detonation-mode plasma current sheet which would very quickly accelerate to the end of the channel. While not yet modeled, one can imagine that the current sheet would transition into a deflagration-mode stationary discharge that would accelerate additional neutral gas as it entered the plasma.

We can further speculate that the Ziemer data ${ }^{5,6}$ may be explained in a manner similar to that presented above for the CHENG thruster. Specifically, during Mode I operation, the accelerator's performance may be detonation modedominated by a current sheet propagating into and entraining neutral gas. However, as the mass bit is lowered the resistance to the current sheet decreases allowing the plasma to reach the end of the accelerator more quickly. If the current sheet transitions to the deflagration mode when it reaches the end of the acceleration channel, we would expect this mode's fractional contribution to the net performance to grow the longer the thruster operates in this mode.

\section{Conclusion}

We have presented a modeling framework to capture the time-evolution of a pulsed plasma accelerator and explored the gas injection and detonation-mode plasma acceleration solutions as a function of several controllable parameters. Gas injection modeling was performed for a variety of mass flow rates, with calculations performed to ensure that conserved properties (mass, momentum) were being conserved in the numerical solution. The detonation mode was modeled while varying the delay time $t_{\text {delay }}$ between the start of gas injection and current sheet initiation, the gas entrainment factor $\lambda_{e}$, and the current sheet mass loss term $\nu_{\text {loss. }}$. We found that the current sheet reached the end of the channel much more quickly when it had less mass to entrain, either because the value of $t_{\text {delay }}$ was small or because $\lambda_{e}$ was reduced. In cases where the current sheet reached the end of the channel more quickly the overall circuit inductance increased more rapidly, reducing the peak discharge current value and leaving a significantly higher voltage on the capacitor at the end of detonation-mode acceleration. The mass loss term did not appear to affect the trajectory much even though the overall mass of the sheet was signficantly reduced. The explanation for this interesting result involved understanding the competing effects of a reduction in the electromagnetic acceleration force and an increase in the neutral gas entrainment drag force, which reduced the overall applied force on the current sheet. The end of the detonation-mode phase of pulsed plasma acceleration forms the basis for the initial conditions of deflagration-mode. Based on the trends observed in the detonation-mode, we can see how modeling the entire detonation-to-deflagration acceleration in a pulsed plasma accelerator might be successful in explaining the operation and performance of all pulsed plasma accelerators.

\section{Acknowledgments}

The authors acknowledge several helpful discussions with J.B. Pearson, A. Martin, T. Moeller, M. LaPointe, M. Glascock, and A. Ratcliffe, which have improved the quality of this work.

\section{References}

\footnotetext{
${ }^{1}$ J. Marshall, "Performance of a Hydromagnetic Plasma Gun," Phys. Fluids, Vol. 3, No. 1, 134-135 (1960).

${ }^{2}$ R.L. Burton, K.E. Clark, and R.G. Jahn, "Measured performance of a multimegawatt MPD thruster," J. Spacecraft Rockets, Vol. 20, No. 3, 299-304 (1983).

${ }^{3}$ K.A. Polzin and C.M. Greve, "Acceleration Modes and Transitions in Pulsed Plasma Accelerators," AIAA Science and Technology (SciTech) Forum, Kissimmee, FL, Jan. 8-12, 2018. AIAA Paper 2018-174.

${ }^{4}$ D.Y. Cheng, "Plasma deflagration and the properties of a coaxial plasma deflagration gun," Nucl. Fusion, Vol. 10, 305-317 (1970).

${ }^{5}$ J.K. Ziemer, "Performance scaling of gas-fed pulsed plasma thrusters," Ph.D. Dissertation 3016-T, Mechanical and Aerospace Engineering, Princeton Univ., Princeton, NJ (2001).

${ }^{6}$ J.K. Ziemer and E.Y. Choueiri, "Scaling laws for electromagnetic pulsed plasma thrusters," Plasma Sources Sci. Technol., Vol. 10, No. 3, 395-405 (2001).

${ }^{7}$ F.R. Poehlmann, M.A. Cappelli, and G.B. Reiker, "Current distribution measurements inside an electromagnetic plasma gun operated in gas-puff mode," Phys. Plasmas, Vol. 17, 123508 (2010).

${ }^{8}$ H. Sitaraman and L.L. Raja, "Magneto-hydrodynamics simulation study of deflagration mode in co-axial plasma accelerators," Phys. Plasmas, Vol. 21, 012104 (2014).
} 